Background In HIV-positive individuals clinicians observe a broad range of skin conditions like xerosis, tumours, rash and drug-induced exanthema as well as common skin infections caused by bacteria, fungi and viruses. Beyond this, some reports point out a higher incidence on atopic conditions like atopic dermatitis (AD), sinusitis, asthma and laboratory findings like hypereosinophilia and Hyper IgE. Methods Between May and November 2006, 196 patients of the HIV outpatient department of the Clinic for Dermatology, Venerology and Allergology at the Ruhr University Bochum underwent a dermatological examination. Skin conditions focusing on $\mathrm{AD}$ were measured by SCORAD (SCORing Atopic Dermatitis) and Erlanger atopy score.

Results In general, 36 patients (18.4\%) out of 196 participants suffered from clinically from $\mathrm{AD}$. Median count at "Erlanger Atopy Score" was 12.8 (median 11.5). Verification by SCORAD showed $55.6 \%(20 / 36)$ with mild, 36.1\% (13/36) with moderate and $8.3 \%$ (3/36) with severe AD. Neither with pruritus and viral load nor with CDC Category a correlation was found. Exclusively CD4 counts were negative correlated with higher Visual analogue scale for prutitus $(p=0.0306)$. Xerosis was diagnosed in more than $53.6 \%$ of the 196 patients and thus was the leading diagnosis, although there was no correlation with the CD4 count, viral load or CDC Category. Furthermore, a negative correlation was found $(p=0.0214)$ between IgE and CD4 and a $p$-value of 0.0111 between IgE and the CDC Category (higher IgE, higher CDC Category) was demonstrated as well.

Conclusion In our sample xerosis cutis was the leading diagnosis. Furthermore, compared to pre-existing literature for the first time standardised diagnostic tools for $\mathrm{AD}$, the SCORAD and the Erlanger Atopy Score, were used to examine HIV-positive individuals. Diagnostic tools help to identify the origin of dry skin in HIV-infected patients and to initiate adequate treatment.

\section{P2.108 AZITHROMYCIN PHARMACOKINETICS AFTER INTRAVENOUS INFUSION IN WOMEN WITH AND WITHOUT PELVIC INFLAMMATORY DISEASES}

doi:10.1136/sextrans-2013-051184.0372

IV V Chebotarev, ${ }^{2} \mathrm{M}$ A Gomberg, ${ }^{3} Y$ A Baykov. 'Stavropol State Medical Academy, Stavropol, Russian Federation; ${ }^{2}$ Moscow Research and Clinical Centre for Dermatovenereology, Moscow, Russian Federation; ${ }^{3}$ Shpakov Central Regional Hospital, Mikhailovsk, Russian Federation

Patients With acute PID are usually treated in hospital, and antibiotics are used intravenously to get the result as soon as possible. Chlamydia trachomatis is a major PID-causing pathogen, and azithromycin is one of the most active antibiotics against this microorganism.

Aim of the study To evaluate azithromycin concentrations after intravenous infusions in tubal tissues from women with and without PID.

Patients and Methods To prevent possible complications after future surgery azithromycin was infused intravenously $(500 \mathrm{mg}$ twice with 24-hours interval prior surgery, total dose $1.0 \mathrm{~g}$ ) into 70 patients with PID (before surgery to prepare them for IVF), and into 28 patients without PID (before surgical sterilisation). Azithromycin pharmacokinetics was studied in tubal tissues incised at surgery $0.5,1,2,3,4,5,6,7,8,9,10,14,16$ and 18 days after the second infusion.

Results In patients without PID maximal azithromycin concentration $(4.30 \pm 0.30 \mu \mathrm{g} / \mathrm{g})$ was achieved 24 hours after infusion and remained constant for 24 hours more, with a steady drop thereafter. In women with PID maximal azithromycin concentration was achieved in tubal tissues 72 hours after the second infusion, and was lower than in women without PID $(3.38 \pm 0.10 \mu \mathrm{g} / \mathrm{g})$. But on 6 th day after infusion azithromycin concentration in inflamed tissues from women with PID was significantly higher than in noninflamed tissues from women without PID $(1.50 \pm 0.10 \mu \mathrm{g} / \mathrm{g}$ and $0.95 \pm 0.15 \mu \mathrm{g} / \mathrm{g}$, respectively). In both groups azithromycin tissue concentration exceeded C. trachomatis MIC $(0.125 \mu \mathrm{g} / \mathrm{g})$ even 18 days after the second infusion.

Conclusion Azithromycin tubal tissue concentration even 18 days after infusion of $1 \mathrm{~g}$ (500 mg twice with 24 hours interval) exceeds MIC to C.trachomatis both in inflamed and non-inflamed tubes. Maximal azithromycin concentration is higher and achieved faster in women without PID, but it is higher in women with PID one week after infusion.

\section{P2.109 SEXUAL TRANSMISSION OF BACTERIAL VAGINOSIS WITHOUT EXPOSURE TO SEMINAL FLUID}

doi:10.1136/sextrans-2013-051184.0373

J R Schwebke, C Muzny. University of Alabama at Birmingham, Birmingham, AL, United States

Background The pathogenesis of BV is poorly characterised although there is considerable evidence that it is sexually transmitted. Some have suggested that the alkalinity of semen may be a factor. We report a case of suspected sexual transmission of BV from a prostatectomized male to a female.

Methods Case report from a prospective study of behavioural factors influencing the vaginal flora wherein women collect daily selfobtained vaginal slides and behavioural data. Slides are Gram stained and interpreted according to Nugent criteria. Women are encouraged to present for evaluation if symptoms occur.

Results A 51 year old female complained of new onset vaginal irritation for one day. She denied discharge, pruritus, or odour and had not recently douched. She was sexually active with one partner, a male who had undergone a radical prostatectomy. Her last unprotected intercourse occurred 3 days prior to onset of symptoms and prior to that she had been abstinent for 6 weeks. A slide obtained the day before her sexual exposure had a Nugent score of 0 . Repeat gramme stain revealed BV with a Nugent score of 8 . Her male partner admitted to unprotected sex 6 days prior to their encounter with another female. A vaginal slide obtained from that partner revealed BV with a Nugent score of 8 .

Conclusion To our knowledge, this is the first report documenting sexual transmission of BV from a male to a female in the absence of semen. The onset of symptoms and her sexual history indicates that the incubation period for BV was 72 hours. It is likely that the patient's male partner became colonised in his distal urethral or coronal sulcus with BV organism(s) after he had unprotected sexual intercourse with his other female partner and transferred those organism(s) on desquamated epithelial cells to our patient during unprotected sex.

\section{P2.110 RISK FACTORS FOR BACTERIAL VAGINOSIS AMONG SYMPTOMATIC WOMEN ATTENDING STI CLINIC IN TEL AVIV, ISRAEL}

doi:10.1136/sextrans-2013-051184.0374

'Z Mor, ${ }^{2} \mathrm{~A}$ Shani, ${ }^{3,2 \mathrm{M}}$ Dan, ${ }^{4,2} \mathrm{~T}$ Shohat. 'Ministry of Health, Ramla, Israel; ${ }^{2} \mathrm{Tel}$ Aviv University, Tel Aviv, Israel; ${ }^{3}$ Wolfson Medical Center, Holon, Israel; ${ }^{4}$ sraeli Center for Disease Control, Tel Hashomer, Israel

Background Bacterial vaginosis (BV) is a common cause for vaginal symptoms and is associated with an increased risk of acquisition of STI/HIV, and with adverse pregnancy outcomes. This study aimed to describe demographic, behavioural and clinical characteristics of symptomatic women diagnosed with BV among those who attended the municipal STI clinic in Tel-Aviv, Israel, and identify risk factors for the disease. 